\title{
Cribriform Carcinoma
}

National Cancer Institute

\section{Source}

National Cancer Institute. Cribriform Carcinoma. NCI Thesaurus. Code C3680.

A carcinoma characterized by the presence of a cribriform architectural pattern.

Representative examples include the intraductal cribriform breast carcinoma and invasive cribriform breast carcinoma. 